\title{
COMPUTATION OF FIXED POINT INDEX AND ITS APPLICATIONS
}

\author{
HUI XING AND JINGXIAN SUN
}

\begin{abstract}
In this paper, we make the nonlinear double integral equation of Hammerstein type the background of the research. Computation for the fixed point index of operators such as $A=K_{1} F_{1} K_{2} F_{2}$ is given. As applications of the main results, we investigate the existence of positive solutions to the nonlinear double integral equation of Hammerstein type and the boundary value problem for the system of elliptic partial differential equations.
\end{abstract}

1. Introduction. Let $E$ be a real Banach space with norm $\|\cdot\|$, and let $P \subset E$ be a cone of $E$. We define a partial ordering $\leq$ with respect to $P$ by $x \leq y$ if and only if $y-x \in P$. $P$ is said to be normal if there exists a positive constant $N$ such that $\theta \leq x \leq y$ implies $\|x\| \leq N\|y\|$, where $\theta$ represents the zero element in $E$ and the smallest $N$ is called the normal constant of $P . P$ is called solid if it contains interior points, i.e., $P \neq \emptyset$. For the concepts and the properties about the cone we refer to $[2,4,17]$.

In this paper, we make the nonlinear double integral equation of Hammerstein type as the background of the research and study the computation of the fixed point index of the operators such as $A=K_{1} F_{1} K_{2} F_{2}$ more systematically. As an application, the following nonlinear double integral equation of Hammerstein type

$$
u(x)=\int_{0}^{1} k_{1}(x, y) f_{1}\left(y, \int_{0}^{1} k_{2}(y, z) f_{2}(z, u(z)) d z\right) d y,
$$

2010 AMS Mathematics subject classification. Primary 57M25, 57M27.

Keywords and phrases. Fixed point index, $\alpha \beta$ homogeneous operators, nonlinear double integral equation of Hammerstein type.

This work is supported by the National Science Foundation of P.R. China (10971179).

The first author is the corresponding author.

Received by the editors on October 12, 2011, and in revised form on July 11, 2013. 
is investigated, where $f_{i}(x, u):[0,1] \times[0,+\infty) \rightarrow[0,+\infty), k_{i}(x, y)$ : $[0,1] \times[0,1] \rightarrow[0,+\infty), i=1,2$. The theory of the fixed point index is one of the main methods in studying the existence of solutions to the nonlinear operator equation. The study of the nonlinear Hammerstein integral equations was initiated by Hammerstein [7] in 1929. Subsequently, a great number of papers dealing with the existence of nontrivial solutions of Hammerstein integral equations have been published, see for example, $[\mathbf{9}, \mathbf{1 0}, \mathbf{1 8}, \mathbf{1 9}, \mathbf{2 0}]$ and the references therein. Yang and O'Regan [20] studied a system of nonlinear Hammerstein integral equations:

$$
\left\{\begin{array}{l}
u(x)=\int_{0}^{1} k(x, y) f(y, u(y), v(y)) d y \\
v(x)=\int_{0}^{1} k(x, y) g(y, u(y), v(y)) d y
\end{array}\right.
$$

The existence of positive solutions of (1.2) was obtained by using topological methods and cone theory. Generally speaking, in order to compute the fixed point index we usually change the systems of differential equations into the nonlinear double integral equation of Hammerstein type, see $[\mathbf{8}, \mathbf{1 4}]$.

In this paper, broader results are obtained when the nonlinear operator is controlled by $\alpha$ homogeneous operator (which is broader than the linear operator). To the best of our knowledge the papers dealing with (1.1) are few when the nonlinear operator is controlled by the $\alpha$ homogeneous operator. Many authors studied computation for the fixed point index when the nonlinear operator is controlled by the homogeneous operator or the linear operator $[\mathbf{5}, \mathbf{8}, \mathbf{1 1}]$, which is different from the results of this paper. Motivated by the work $[5,7,8,11,14,15,18,3,19,20]$, we obtained the results of this paper.

The organization of this paper is as follows. In Section 2, some useful preliminaries are presented. In Section 3, we give the results of computation of the fixed point index of the operators such as $A=K_{1} F_{1} K_{2} F_{2}$ more systematically. In Sections 4 and 5 , we use the main results of Section 3 to establish the existence of positive solutions to the nonlinear double integral equation of Hammerstein type and the positive solutions of the system of elliptic partial differential equations. 
2. Preliminaries. Suppose that $X$ is a retract in Banach space $E$ and $U$ is a bounded open set in $X$. Let $A: \bar{U} \rightarrow X$ be a completely continuous operator, which has no fixed point on $\partial U$ (the boundary of $U$ with respect to $X)$. Then we can define the fixed point index $i(A, U, X)$ of $A$ over $U$ with respect to $X$. (For example, every closed convex subset is a retract.) One can refer to [4] for the definition and properties of $i(A, U, X)$.

\subsection{Definitions.}

Definition 2.1. [13]. Let $P$ be a solid cone of $E$. The operator $A: P \rightarrow P$ is a positive $\alpha$ homogeneous operator, if $\mathrm{A}$ satisfies $A(t x)=t^{\alpha} A x$, for any $t>0$ and $\alpha \in R^{+}$. In particular, when $\alpha=1$, the operator $\mathrm{A}$ is called homogeneous operator.

Definition 2.2. [1]. Let $P$ be a cone of $E$. The operator $T: E \rightarrow E$ is called e-positive if there exists an element $e \in P \backslash\{\theta\}$, such that, for every $u \in P \backslash\{\theta\}$, there are numbers $\alpha=\alpha(u), \beta=\beta(u)>0$, such that

$$
\alpha e<T u<\beta e .
$$

\subsection{Lemmas.}

Lemma 2.3. [12]. Let $a, b$ be real numbers. Then

(i) $|a+b|^{\mu} \leq|a|^{\mu}+|b|^{\mu}$, for $0 \leq \mu \leq 1$;

(ii) $|a+b|^{\mu} \leq 2^{\mu-1}\left(|a|^{\mu}+|b|^{\mu}\right)$, for $\mu>1$.

Lemma 2.4. [17]. Let $\Omega$ be a bounded open set of $E$ with $\theta \in \Omega$. Assume that $A: P \cap \bar{\Omega} \rightarrow P$ is a completely continuous operator satisfying

$$
A x \neq \mu x, \quad x \in P \cap \partial \Omega, \mu \geq 1 .
$$

Then we have $i(A, \Omega \cap P, P)=1$.

Lemma 2.5. [6]. Let $\Omega \subset E$ be a bounded open set with $\theta \in \Omega$. Suppose $A: \bar{\Omega} \cap P \rightarrow P$ is a completely continuous operator and has no fixed points on $\partial \Omega \cap P$. If $\|A w\| \leq\|w\|$, for all $w \in \partial \Omega \cap P$, then $i(A, \Omega \cap P, P)=1$. 
Lemma 2.6. [17]. Suppose that $A: P \cap \bar{\Omega} \rightarrow P$ is a completely continuous operator. If there exist $u^{*} \in P$ and $u^{*} \neq \theta$ such that

$$
x-A x \neq \lambda u^{*}, \quad x \in P \cap \partial \Omega, \lambda \geq 0,
$$

then we have $i(A, \Omega \cap P, P)=0$.

Lemma 2.7. [20]. If $p: R^{+} \rightarrow R^{+}$is concave, then $p(a+b) \leq$ $p(a)+p(b)$, for $a \in R^{+}$and $b \in R^{+}$.

3. Main results. In order to compute the fixed point index of operators such as $A=K_{1} F_{1} K_{2} F_{2}$, here we list the hypotheses to be used later:

(H) Let $K_{1}, K_{2}: P \rightarrow P$ be positive linear operators, $F_{1}, F_{2}: P \rightarrow P$ nonlinear operators, and $A=K_{1} F_{1} K_{2} F_{2}$ a completely continuous operator.

Theorem 3.1. Assume that condition $(\mathrm{H})$ holds. Let $P$ be a normal cone in $E$, the normal constant of $P$ equal to 1 . Suppose that $G_{1}$ : $P \rightarrow P$ is a positive $\alpha$ homogeneous increasing operator with $0<\alpha \leq 1$ and $G_{2}: P \rightarrow P$ is a positive $\beta$ homogeneous increasing operator with $0<\alpha \beta \leq 1$. If there exists $w_{i}>\theta, i=1,2$, such that:

(i) $F_{i} x \leq G_{i} x+w_{i}$, for all $x \in P$;

(ii) $G_{1}(x+y) \leq G_{1} x+G_{1} y$, for all $x, y \in P$;

(iii) $C=K_{1} G_{1} K_{2} G_{2}$, and for all $x \in P \backslash\{\theta\}$,

$$
\sup _{\|x\|=1}\|C x\|<\left\{\begin{array}{l}
\infty, \quad 0<\alpha \beta<1 \\
1, \quad \alpha \beta=1,
\end{array}\right.
$$

then $i\left(A, P \cap T_{R}, P\right)=1$, where $T_{R}=\{x \in E:\|x\| \leq R\}$ for sufficiently large $R$.

Remark. Let $T_{R}=\{x \in E:\|x\| \leq R\}$ be a closed ball of center $\theta$ and radius $R$. For sufficiently large $R \geq R_{0}$, Theorem 3.1 holds, where $R_{0}$ is defined in the following proof. 
Proof. By Definition 2.1, we have $G_{1}(t x)=t^{\alpha} G_{1} x, G_{2}(t x)=t^{\beta} G_{2} x$, and

$$
\begin{aligned}
C(t x) & =K_{1} G_{1} K_{2} G_{2}(t x)=K_{1} G_{1} K_{2} t^{\beta} G_{2} x \\
& =K_{1} G_{1} t^{\beta}\left(K_{2} G_{2} x\right)=K_{1}\left(t^{\beta}\right)^{\alpha} G_{1} K_{2} G_{2} x \\
& =\left(t^{\beta}\right)^{\alpha} K_{1} G_{1} K_{2} G_{2} x=t^{\alpha \beta} K_{1} G_{1} K_{2} G_{2} x \\
& =t^{\alpha \beta} C x .
\end{aligned}
$$

When $0<\alpha \beta<1, C$ is an $\alpha \beta$ homogeneous operator. By condition (iii), there exists a constant $M$ such that $\sup _{\|x\|=1}\|C x\| /\|x\|<M$. Letting $0<\delta<\min \{1, M\}$, we have $M / \delta>1$. For sufficiently large $t>(M / \delta)^{1 /(1-\alpha \beta)}$, by $(3.2)$, we have

$$
\sup _{\|x\|=1} \frac{\|C t x\|}{\|t x\|}=t^{\alpha \beta-1} \cdot \sup _{\|x\|=1} \frac{\|C x\|}{\|x\|}<t^{\alpha \beta-1} \cdot M<\delta .
$$

Letting $u=t x$ in (3.3), when $t$ is large enough, we have

$$
\sup _{u \in P \backslash\{\theta\}} \frac{\|C u\|}{\|u\|}<\delta
$$

When $\alpha \beta=1, C$ is a homogeneous operator. By condition (iii), we have $\sup _{\|x\|=1}\|C x\| /\|x\|<1$. It follows from (3.2) that

$$
\sup _{\|x\|=1} \frac{\|C s x\|}{\|s x\|}=\sup _{\|x\|=1} \frac{s\|C x\|}{s\|x\|}<1 .
$$

In (3.5), when $s$ is large enough, letting $u=s x$, then

$$
\sup _{u \in P \backslash\{\theta\}} \frac{\|C u\|}{\|u\|}<1 .
$$

By conditions (i), (ii) and (iii), we have

$$
\begin{aligned}
A x & =K_{1} F_{1} K_{2} F_{2} x \leq K_{1} G_{1} K_{2} F_{2} x+K_{1} w_{1} \\
& \leq K_{1} G_{1} K_{2}\left(G_{2} x+w_{2}\right)+K_{1} w_{1} \\
& =K_{1} G_{1}\left(K_{2} G_{2} x+K_{2} w_{2}\right)+K_{1} w_{1} \\
& \leq K_{1} G_{1} K_{2} G_{2} x+K_{1} G_{1} K_{2} w_{2}+K_{1} w_{1} \\
& =C x+w_{0}
\end{aligned}
$$


where $w_{0}=K_{1} G_{1} K_{2} w_{2}+K_{1} w_{1}$. Select

$$
R_{0}>\frac{\left\|w_{0}\right\|}{1-\delta}
$$

Let

$$
T_{R_{0}}=\left\{x \in E:\|x\|<R_{0}\right\} .
$$

Set $H(t, u)=u-t A u$. Next, we prove

$$
H(t, u)=u-t A u \neq \theta, \quad \text { for all } t \in[0,1], \quad u \in \partial T_{R_{0}} \cap P .
$$

Assume on the contrary that there exists a $u_{0} \in \partial T_{R_{0}} \cap P$ with $\left\|u_{0}\right\|=R_{0}$ and $t_{0} \in[0,1]$ such that $u_{0}-t_{0} A u_{0}=\theta$. For $0<\alpha \beta<1$, by (3.7), we have

$$
u_{0} \leq t_{0} C u_{0}+t_{0} w_{0} \leq C u_{0}+w_{0},
$$

since $P$ is the normal cone with normal constant 1. By (3.4) and (3.9), we have

$$
\left\|u_{0}\right\| \leq\left\|C u_{0}\right\|+\left\|w_{0}\right\| \leq \delta\left\|u_{0}\right\|+\left\|w_{0}\right\| .
$$

By (3.10), we have $\left\|u_{0}\right\| \leq\left\|w_{0}\right\| /(1-\delta)$, which contradicts $\left\|u_{0}\right\|=$ $R_{0}>\left\|w_{0}\right\| /(1-\delta)$. By Lemma 2.4 , for sufficiently large $R>R_{0}$, we have $i\left(A, P \cap T_{R}, P\right)=1$. When $\alpha \beta=1$, since $P$ is a normal cone with the normal constant 1 , by (3.6) and (3.7), we have

$$
\|A u\| \leq\|C u\|+\left\|w_{0}\right\| \leq\|u\| .
$$

For sufficiently large $R \geq\|u\| \geq\|A u\|$, by Lemma 2.5, we have $i\left(A, P \cap T_{R}, P\right)=1$. The proof has been completed.

Corollary 3.2. Let $P$ be a normal cone of $E$, the normal constant of $P$ equal to 1 and $A: P \rightarrow P$ a completely continuous operator. If there exists a positive $\alpha$ homogeneous increasing operator $B: P \rightarrow P$ with $0<\alpha \leq 1$ and there exists $u_{0} \in P$ such that:

(i) $A x \leq B x+u_{0}$, for all $x \in P$;

(ii) for all $x \in P \backslash\{\theta\}$,

$$
\sup _{\|x\|=1}\|B x\|< \begin{cases}\infty, & 0<\alpha \beta<1, \\ 1, & \alpha \beta=1,\end{cases}
$$


then $i\left(A, P \cap T_{R}, P\right)=1$, where $T_{R}=\{x \in E:\|x\| \leq R\}$ for sufficiently large $R$.

Proof. In the proof of Theorem 3.1, set $C=B, w_{0}=u_{0}$, which completes the proof.

Theorem 3.3. Assume that condition $(\mathrm{H})$ holds and the operator $A$ has no fixed point on $P \cap \partial T_{r}$ for $r>0$, where $T_{r}=\{x \in E:\|x\| \leq r\}$. Suppose that $G_{1}: P \rightarrow P$ is a positive $\alpha$ homogeneous increasing operator and $G_{2}: P \rightarrow P$ is a positive $\beta$ homogeneous increasing operator such that:

(i) $F_{i} x \geq G_{i} x, i=1,2$, for all $x \in P \cap \bar{T}_{r}$;

(ii) for $\alpha \beta \in(0,1)$, there exist $u^{*} \in P \backslash\{\theta\}$ and a real number $\delta>0$ such that $C u^{*} \geq \delta u^{*}$, where $C=K_{1} G_{1} K_{2} G_{2}$,

then $i\left(A, P \cap T_{r}, P\right)=0$ for sufficiently small $r$.

Remark. Let $T_{r}=\{x \in E:\|x\| \leq r\}$ be a closed ball of center $\theta$ and radius $r$. For $0<r \leq r_{0}$, Theorem 3.3 holds, where $r_{0}$ is introduced in the following proof.

Proof. We divide the proof into two steps.

Step 1: It follows from (3.2) that $C$ is a positive $\alpha \beta$ homogeneous operator. By conditions (i) and (ii), we have

$$
A x=K_{1} F_{1} K_{2} F_{2} x \geq K_{1} F_{1} K_{2} G_{2} x \geq K_{1} G_{1} K_{2} G_{2} x=C x .
$$

Step 2: By condition (ii), when $\alpha \beta<1, C u^{*} \geq \delta u^{*}$. When $0<\delta<1, \delta^{1 /(1-\alpha \beta)}<1$ is obvious. Let $0<t<\delta^{1 /(1-\alpha \beta)}$, we have $C t u^{*}=t^{\alpha \beta} C u^{*} \geq t^{\alpha \beta} \delta u^{*} \geq t u^{*}$. Setting $t u^{*}=v^{*}$, we have $C v^{*} \geq v^{*}$. When $\delta \geq 1$, we have $C u^{*} \geq \delta u^{*} \geq u^{*}$. Setting $u^{*}=v^{*}$, we have $C v^{*} \geq v^{*}$. Since $u^{*} \in P \backslash\{\theta\}$, when $\delta>0$, there exists a $v^{*} \in P \backslash\{\theta\}$ such that $C v^{*} \geq v^{*}$. Letting $D=\left\{u \in E: u \geq v^{*}\right\}$, we have $d=d(\theta, D)>0$. When $0<r_{0}<d$, for $x \in P,\|x\| \leq r_{0}$, we have that $x \notin D$ and $x \nsupseteq v^{*}$. Let $T_{r_{0}}=\left\{x \in E:\|x\| \leq r_{0}\right\}$. Next, we prove

$$
x-A x \neq \lambda v^{*}, \quad \text { for all } x \in P \cap \partial T_{r_{0}}, \lambda \geq 0 \text {. }
$$


We assume on the contrary that there exists $x_{0} \in P \cap \partial T_{r_{0}}$ with $\lambda_{0}>0$ (since $A$ has no fixed point on $P \cap \partial T_{r_{0}}$ ), such that

$$
x_{0}-A x_{0}=\lambda_{0} v^{*} .
$$

By (3.14), we have $x_{0}=A x_{0}+\lambda_{0} v^{*} \geq \lambda_{0} v^{*}$. Setting $\lambda^{*}=\sup \left\{\lambda: x_{0} \geq\right.$ $\left.\lambda v^{*}\right\}$, we have that $\lambda^{*} \geq \lambda_{0}>0$ and $x_{0} \geq \lambda^{*} v^{*}$. Since $x_{0} \nsucceq v^{*}$, we have $0<\lambda^{*}<1$. By (3.12) and (3.14), we have $x_{0} \geq A x_{0} \geq C x_{0}$. $C$ is an increasing operator, therefore, $C x_{0} \geq C \lambda^{*} v^{*}$. Since $C$ is a positive $\alpha \beta$ homogeneous operator and $0<\alpha \beta<1$, we have

$$
\begin{aligned}
x_{0} & =A x_{0}+\lambda_{0} v^{*} \geq C x_{0}+\lambda_{0} v^{*} \\
& \geq C \lambda^{*} v^{*}+\lambda_{0} v^{*}=\left(\lambda^{*}\right)^{\alpha \beta} C v^{*}+\lambda_{0} v^{*} \\
& \geq \lambda^{*} C v^{*}+\lambda_{0} v^{*} \geq \lambda^{*} v^{*}+\lambda_{0} v^{*} \\
& =\left(\lambda^{*}+\lambda_{0}\right) v^{*} .
\end{aligned}
$$

This contradicts the above definition of $\lambda^{*}$. For sufficiently small $r$ such that $0<r<r_{0}$, we have $i\left(A, P \cap T_{r}, P\right)=0$.

Theorem 3.4. In Theorem 3.3, condition (ii) is substituted for the following condition:

(ii') for $\alpha \beta=1$, there exists $u^{*} \in P \backslash\{\theta\}$ such that $C u^{*} \geq u^{*}$, where $C=K_{1} G_{1} K_{2} G_{2}$.

Then the conclusion of Theorem 3.3 still holds.

Proof. It follows from the proof of Theorem 3.3 that $A x \geq C x$. By condition ( ii $^{\prime}$ ), when $\alpha \beta=1, C$ is a positive homogeneous operator with $C u^{*} \geq u^{*}$. Let $T_{r}=\{x \in E:\|x\| \leq r\}$. Next, we prove

$$
x-A x \neq \lambda u^{*}, \quad x \in P \cap \partial T_{r}, \lambda \geq 0 .
$$

By the same argument as Step 2 of the proof of Theorem 3.3, we have $i\left(A, P \cap T_{r}, P\right)=0$.

Corollary 3.5. Let $\Omega$ be a bounded open set in $E$ with $\theta \in \Omega$ and $A: P \cap \bar{\Omega} \rightarrow P$ a completely continuous operator. If there exist another Banach space $E_{1}$, a cone $P_{1}$ in $E_{1}$, a positive $\alpha$ homogeneous operator $B: P \rightarrow P$ with $0<\alpha<1$, a linear operator $N: P \rightarrow P_{1}$, an increasing operator $L: P_{1} \rightarrow P_{1}$ and a real number $\delta>0$ such that: 
(i) $N A x \geq N B x$, for all $x \in P \cap \bar{\Omega}$;

(ii) there exist $u^{*} \in P \backslash\{\theta\}$ and a non-negative integer $n$ such that $N B^{n} u^{*} \geq \delta N u^{*}, N u^{*} \neq \theta$

(iii) $N B x \equiv L N x$, for all $x \in P$;

(iv) $A$ has no fixed point on $P \cap \partial T_{r}$.

Then $i\left(A, P \cap T_{r}, P\right)=0$, where $T_{r}=\{x \in E:\|x\| \leq r\}$ for sufficiently small $r$.

Remark. Let $T_{r}=\{x \in E:\|x\| \leq r\}$ be a closed ball of center $\theta$ and radius $r$. For sufficiently small $r>0$ and $r<r_{0}$, Theorem 3.3 holds, where $r_{0}$ is introduced in the following proof.

Proof. Since $B$ is a positive $\alpha(0<\alpha<1)$ homogeneous operator, it follows from $0<\delta<1$ that $\delta^{1 /\left(1-\alpha^{n}\right)}<1$. Setting $0<t<\delta^{1 /\left(1-\alpha^{n}\right)}$, by condition (ii), we have

$$
\begin{aligned}
N B^{n} t u^{*} & =N B^{n-1} t^{\alpha} B u^{*} \\
& =N B^{n-2} t^{\alpha^{2}} B^{2} u^{*}=N t^{\alpha^{n}} B^{n} u^{*} \\
& =t^{\alpha^{n}} N B^{n} u^{*} \geq t^{\alpha^{n}} N \delta u^{*} \\
& \geq t N u^{*}=N t u^{*} .
\end{aligned}
$$

Set $t u^{*}=v^{*}$. By (3.17), we have $N B^{n} v^{*} \geq N v^{*}$. When $\delta \geq 1$, $N B^{n} u^{*} \geq N \delta u^{*} \geq N u^{*}$. Set $u^{*}=v^{*}$, we have $N B^{n} v^{*} \geq N v^{*}$. Since $u^{*} \in P \backslash\{\theta\}$, when $\delta>0$, there exists $v^{*} \in P \backslash\{\theta\}$, such that $N B^{n} v^{*} \geq N v^{*}$. Let $D=\left\{u \in E: u \geq v^{*}\right\}$ and $d=d(\theta, D)>0$. When $0<r_{0}<d, x \in P,\|x\| \leq r_{0}$, we have that $x \notin D$; hence, $x \geq v^{*}$.

Next, we will prove

$$
x-A x \neq \lambda v^{*}, \quad \text { for all } x \in P \cap \partial T_{r_{0}}, \lambda \geq 0 .
$$

We assume on the contrary that there exist $x_{0} \in P \cap \partial T_{r_{0}}$ and $\lambda_{0}>0$ (since $A$ has no fixed point on $P \cap \partial T_{r_{0}}$ ) such that

$$
x_{0}-A x_{0}=\lambda_{0} v^{*} .
$$

By (3.19), we have $N x_{0}=N A x_{0}+\lambda_{0} N v^{*} \geq \lambda_{0} N v^{*}$. Letting $\lambda^{*}=\sup \left\{\lambda: N x_{0} \geq \lambda N v^{*}\right\}$, it is evident that $\lambda^{*} \geq \lambda_{0}>0$ and $N x_{0} \geq \lambda^{*} N v^{*}$. Since $x_{0} \geq v^{*}$ and $N x_{0} \geq N v^{*}$, we have $0<\lambda^{*}<1$. 
By condition (i) and (3.19), we have $N x_{0} \geq N A x_{0} \geq N B x_{0}$. By condition (iii), we have

$$
N B x_{0}=L N x_{0} \geq L N B x_{0} \geq N B^{2} x_{0} .
$$

For any non-negative integer $i$, by (3.20) we have $N B^{i} x_{0} \geq N B^{i+1} x_{0}$. Therefore, $N B x_{0} \geq N B^{n} x_{0}$. By condition (iii), for $x \in P$, we have $N B^{n} x=L^{n} N x$. Since $B$ is a positive $\alpha$ homogeneous operator with $0<\alpha<1$, we have $0<\alpha^{n}<1$ and $N B^{n} v^{*} \geq N v^{*}$. Hence, we have

$$
\begin{aligned}
N x_{0} & =N A x_{0}+\lambda_{0} N v^{*} \geq N B x_{0}+\lambda_{0} N v^{*} \\
& \geq N B^{n} x_{0}+\lambda_{0} N v^{*}=L^{n} N x_{0}+\lambda_{0} N v^{*} \\
& \geq L^{n} N \lambda^{*} v^{*}+\lambda_{0} N v^{*}=N B^{n} \lambda^{*}+\lambda_{0} N v^{*} \\
& =\left(\lambda^{*}\right)^{\alpha^{n}} N B^{n} v^{*}+\lambda_{0} N v^{*} \geq \lambda^{*} N B^{n} v^{*}+\lambda_{0} N v^{*} \\
& \geq \lambda^{*} N v^{*}+\lambda_{0} N v^{*}=\left(\lambda^{*}+\lambda_{0}\right) N v^{*} .
\end{aligned}
$$

This contradicts the above definition of $\lambda^{*}$. For sufficiently small $r \leq r_{0}$, by Lemma 2.6, we have $i\left(A, P \cap T_{r}, P\right)=0$.

Corollary 3.6. Let $\Omega$ be a bounded open set in $E$ with $\theta \in \Omega$ and $A: P \cap \bar{\Omega} \rightarrow P$ a completely continuous operator. If there exist a positive $\alpha$ homogeneous increasing operator $B: P \rightarrow P$ with $0<\alpha<1$, $u^{*} \in P \backslash\{\theta\}$ and real number $\delta>0$ such that:

(i) $A x \geq B x$, for all $x \in P \cap \bar{\Omega}$;

(ii) $B u^{*} \geq \delta u^{*}$;

(iii) $A$ has no fixed points on $P \cap \partial T_{r}$, where $T_{r}=\{x \in E:\|x\| \leq r\}$.

Then $i\left(A, P \cap T_{r}, P\right)=0$ for sufficiently small $r$.

Proof. In the proof of Theorem 3.3, let $C=B$, which completes the proof.

4. The application for the nonlinear double integral equation of Hammerstein type. Next, we use the main results of Section 3 to study the nonlinear double integral equation of Hammerstein type

$$
u(x)=\int_{0}^{1} k_{1}(x, y) f_{1}\left(y, \int_{0}^{1} k_{2}(y, z) f_{2}(z, u(z)) d z\right) d y=A u(x) .
$$


Let $E=C[0,1], f_{i}(x, u):[0,1] \times[0,+\infty) \rightarrow[0,+\infty), k_{i}(x, y):$ $[0,1] \times[0,1] \rightarrow[0,+\infty), i=1,2 . P=\{u \in C[0,1]: u(x) \geq 0$, for all $x \in[0,1]\}$. Next we investigate the existence of the solution to the nonlinear integral equation (4.1). For convenience, we make the following assumptions:

$\left(H_{1}\right)$ There exist $\alpha \in(0,1]$ and $\beta \in(0, \infty)$ satisfying $\alpha \beta \in(0,1)$, $a_{1}(x)>0, a_{2}(x)>0, b_{1}(x) \geq 0, b_{2}(x) \geq 0$ and $a_{1}(x), a_{2}(x), b_{1}(x)$ and $b_{2}(x) \in C[0,1]$ such that

$$
\begin{aligned}
& f_{1}(x, u) \leq a_{1}(x) u^{\alpha}+b_{1}(x), \quad \text { for all } x \in[0,1], u \geq 0, \\
& f_{2}(x, u) \leq a_{2}(x) u^{\beta}+b_{2}(x), \quad \text { for all } x \in[0,1], u \geq 0 .
\end{aligned}
$$

We define the Nemytskii operators $G_{1}, G_{2}: P \rightarrow P$, which are determined by $u^{\alpha}$ and $u^{\beta}$ respectively, and

$$
\begin{array}{ll}
G_{1} u^{\alpha}(x)=g_{1}\left(x, u^{\alpha}(x)\right), & x \in[0,1], \\
G_{2} u^{\beta}(x)=g_{2}\left(x, u^{\beta}(x)\right), & x \in[0,1],
\end{array}
$$

where $g_{1}, g_{2}:[0,1] \times[0,+\infty) \rightarrow[0,+\infty)$.

$\left(H_{2}\right)$ There exist $\alpha_{1} \in(0,+\infty), d_{1}(x), d_{2}(x) \in C[0,1]$ and $d_{1}(x) \geq 0$, $d_{2}(x) \geq 0$ such that

$$
\begin{array}{ll}
f_{1}(x, u) \geq d_{1}(x) u^{\alpha_{1}}, & \text { for all } x \in[0,1], 0 \leq u \leq s, \\
f_{2}(x, u) \geq d_{2}(x) u^{1 / \alpha_{1}}, & \text { for all } x \in[0,1], 0 \leq u \leq r,
\end{array}
$$

where $r$ and $s$ are sufficiently small positive constants with $s \leq r$ as long as the operator $A$ has no fixed point on $P \cap \partial T_{r}$ for $r>0$, where $T_{r}=\{x \in E:\|x\| \leq r\}$.

$\left(H_{3}\right)$ There exist $\alpha_{2} \in(0,+\infty), \alpha_{2} \beta_{2} \in(0,1), m_{1}(x), m_{2}(x) \in$ $C[0,1]$ and continuous functions $m_{1}(x) \geq 0$ and $m_{2}(x) \geq 0$ such that

$$
\begin{aligned}
& f_{1}(x, u) \geq m_{1}(x) u^{\alpha_{2}}, \quad \text { for all } x \in[0,1], 0 \leq u \leq s, \\
& f_{2}(x, u) \geq m_{2}(x) u^{\beta_{2}}, \quad \text { for all } x \in[0,1], 0 \leq u \leq r,
\end{aligned}
$$

where $r$ and $s$ are sufficiently small positive constants, and $0<s \leq$ $r<d=d(\theta, D)$. Here $D=\left\{u \in E: u \geq v^{*}=v^{*}\left(\alpha_{2}, \beta_{2}, \delta, u^{*}\right), v^{*} \in\right.$ $P \backslash\{\theta\}\}$ and $\delta, u^{*}$ are defined in the following condition $\left(H_{5}\right)$. 
$\left(H_{4}\right)$ There exists $u^{*} \in P \backslash\{\theta\}$ such that $B_{1} u^{*} \geq u^{*}$, where $B_{1}$ is a homogeneous operator

$$
B_{1} u(x)=\int_{0}^{1} k_{1}(x, y) d_{1}(y)\left(\int_{0}^{1} k_{2}(y, z) d_{2}(z) u^{1 / \alpha_{1}}(z) d z\right)^{\alpha_{1}} d y .
$$

$\left(H_{5}\right)$ There exists $u^{*} \in P \backslash\{\theta\}$ and real number $\delta>0$ such that $B_{2} u^{*} \geq \delta u^{*}$, where $B_{2}$ is a $\alpha_{2} \beta_{2}$ homogeneous operator

$$
B_{2} u(x)=\int_{0}^{1} k_{1}(x, y) m_{1}(y)\left(\int_{0}^{1} k_{2}(y, z) m_{2}(z) u^{\beta_{2}}(z) d z\right)^{\alpha_{2}} d y .
$$

Theorem 4.1. Suppose that $\left(H_{1}\right),\left(H_{2}\right)$ and $\left(H_{4}\right)$ are satisfied. Then equation (4.1) has at least one positive solution.

Proof. It is evident that $A: P \rightarrow P$ is completely continuous. We divide the proof into the following two steps.

Step 1: By $\left(H_{1}\right)$, Lemma $2.3(1)$ and Lemma 2.7, we have

$$
\begin{aligned}
A u(x)= & \int_{0}^{1} k_{1}(x, y) f_{1}\left(y, \int_{0}^{1} k_{2}(y, z) f_{2}(z, u(z)) d z\right) d y \\
\leq & \int_{0}^{1} k_{1}(x, y)\left(a_{1}(y)\left(\int_{0}^{1} k_{2}(y, z) f_{2}(z, u(z)) d z\right)^{\alpha}+b_{1}(y)\right) d y \\
= & \int_{0}^{1} k_{1}(x, y) a_{1}(y)\left(\int_{0}^{1} k_{2}(y, z) f_{2}(z, u(z)) d z\right)^{\alpha} d y \\
& \left.+\int_{0}^{1} k_{1}(x, y) b_{1}(y)\right) d y \\
\leq & \int_{0}^{1} k_{1}(x, y) a_{1}(y)\left(\int_{0}^{1} k_{2}(y, z)\left(a_{2}(z) u^{\beta}(z)+b_{2}(z)\right) d z\right)^{\alpha} d y \\
& +\int_{0}^{1} k_{1}(x, y) b_{1}(y) d y \\
= & \int_{0}^{1} k_{1}(x, y) a_{1}(y)\left(\int_{0}^{1} k_{2}(y, z)\left(a_{2}(z) u^{\beta}(z)+b_{2}(z)\right) d z\right)^{\alpha} d y \\
& +\int_{0}^{1} k_{1}(x, y) b_{1}(y) d y \\
\leq & \int_{0}^{1} k_{1}(x, y) a_{1}(y)\left(\int_{0}^{1} k_{2}(y, z) a_{2}(z) u^{\beta}(z) d z\right)^{\alpha} d y
\end{aligned}
$$




$$
\begin{aligned}
& +\int_{0}^{1} k_{1}(x, y) a_{1}(y)\left(\int_{0}^{1} k_{2}(y, z) b_{2}(z) d z\right)^{\alpha} d y \\
& +\int_{0}^{1} k_{1}(x, y) b_{1}(y) d y \\
& =C u(x)+u_{0},
\end{aligned}
$$

where

$u_{0}=\int_{0}^{1} k_{1}(x, y) a_{1}(y)\left(\int_{0}^{1} k_{2}(y, z) b_{2}(z) d z\right)^{\alpha} d y+\int_{0}^{1} k_{1}(x, y) b_{1}(y) d y$

$C$ is an $\alpha \beta$ homogeneous operator with the following form:

$$
C u(x)=\int_{0}^{1} k_{1}(x, y) a_{1}(y)\left(\int_{0}^{1} k_{2}(y, z) a_{2}(z) u^{\beta}(z) d z\right)^{\alpha} d y
$$

and

$$
\begin{aligned}
G_{1}\left(K_{2} G_{2} u(y)+K_{2} w_{2}\right)= & a_{1}(y)\left(\int_{0}^{1} k_{2}(y, z)\left(a_{2}(z) u^{\beta}(z)+b_{2}(z)\right) d z\right)^{\alpha} \\
\leq & a_{1}(y)\left(\int_{0}^{1} k_{2}(y, z) a_{2}(z) u^{\beta}(z) d z\right)^{\alpha} \\
& +a_{1}(y)\left(\int_{0}^{1} k_{2}(y, z) b_{2}(z) d z\right)^{\alpha} \\
= & G_{1} K_{2} G_{2} u(y)+G_{1} K_{2} w_{2} .
\end{aligned}
$$

By (4.3), we have

$$
\begin{aligned}
\|C u\| & =\max _{x \in[0,1]}|C u(x)| \\
& =\max _{x \in[0,1]}\left|\int_{0}^{1} k_{1}(x, y) a_{1}(y)\left(\int_{0}^{1} k_{2}(y, z) a_{2}(z) u^{\beta}(z) d z\right)^{\alpha} d y\right| .
\end{aligned}
$$

It is evident that $\sup _{\|u\|=1}\|C u\|<+\infty$. It follows from (4.2), (4.4) and (4.5) that conditions (i), (ii) and (iii) of Theorem 3.1 are satisfied. Therefore, $i\left(A, P \cap T_{R}, P\right)=1$.

Step 2: By $\left(H_{2}\right)$, there exists $r>0$, for $0 \leq u \leq r$ and every $y \in[0,1]$ such that $\int_{0}^{1} k_{2}(y, z) f_{2}(z, u(z)) d z \leq s$ and the operator $A$ has no fixed 
point on $P \cap \partial T_{r}$, we have

$$
\begin{aligned}
A u(x) & =\int_{0}^{1} k_{1}(x, y) f_{1}\left(y, \int_{0}^{1} k_{2}(y, z) f_{2}(z, u(z)) d z\right) d y \\
& \geq \int_{0}^{1} k_{1}(x, y) d_{1}(y)\left(\int_{0}^{1} k_{2}(y, z) f_{2}(z, u(z)) d z\right)^{\alpha_{1}} d y \\
& \geq \int_{0}^{1} k_{1}(x, y) d_{1}(y)\left(\int_{0}^{1} k_{2}(y, z) d_{2}(z) u^{1 / \alpha_{1}}(z) d z\right)^{\alpha_{1}} d y \\
& =B_{1} u(x),
\end{aligned}
$$

where

$$
B_{1} u(x)=\int_{0}^{1} k_{1}(x, y) d_{1}(y)\left(\int_{0}^{1} k_{2}(y, z) d_{2}(z) u^{1 / \alpha_{1}}(z) d z\right)^{\alpha_{1}} d y
$$

It is evident that $B_{1}$ is a homogeneous operator. By (4.6) and condition $\left(H_{4}\right)$, the conditions of Theorem 3.4 are satisfied. Therefore, $i(A, P \cap$ $\left.T_{r}, P\right)=0$. Applying the additivity of the fixed point index, we have

$$
i\left(A,\left(T_{R} \backslash \bar{T}_{r}\right) \cap P, P\right)=i\left(A, T_{R} \cap P, P\right)-i\left(A, \bar{T}_{r} \cap P, P\right)=1 .
$$

Hence, equation (4.1) has at least one positive solution.

Theorem 4.2. Suppose that $\left(H_{1}\right),\left(H_{3}\right)$ and $\left(H_{5}\right)$ are satisfied. Then equation (4.1) has at least one positive solution.

Proof. We divide the proof into the following two steps.

Step 1: Step 1 of the proof of Theorem 4.1 still holds.

Step 2: By $\left(H_{3}\right)$ and $\left(H_{5}\right)$, there exist $r>0$ and $v^{*} \in P \backslash\{\theta\}$ for $0 \leq u \leq r<d=d(\theta, D)$ and every $y \in[0,1]$ such that $\int_{0}^{1} k_{2}(y, z) f_{2}(z, u(z)) d z \leq s$. We have

$$
\begin{aligned}
A u(x) & =\int_{0}^{1} k_{1}(x, y) f_{1}\left(y, \int_{0}^{1} k_{2}(y, z) f_{2}(z, u(z)) d z\right) d y \\
& \geq \int_{0}^{1} k_{1}(x, y) m_{1}(y)\left(\int_{0}^{1} k_{2}(y, z) f_{2}(z, u(z)) d z\right)^{\alpha_{2}} d y \\
& \geq \int_{0}^{1} k_{1}(x, y) m_{1}(y)\left(\int_{0}^{1} k_{2}(y, z) m_{2}(z) u^{\beta_{2}}(z) d z\right)^{\alpha_{2}} d y \\
& =B_{2} u(x) .
\end{aligned}
$$




$$
B_{2} u(x)=\int_{0}^{1} k_{1}(x, y) m_{1}(y)\left(\int_{0}^{1} k_{2}(y, z) m_{2} u^{\beta_{2}}(z) d z\right)^{\alpha_{2}} d y
$$

$B_{2}$ is an $\alpha_{2} \beta_{2}$ homogeneous operator. By (4.7) and $\left(H_{5}\right)$, the conditions of Theorem 3.3 are satisfied. Letting $T_{r}=\{x \in E:\|x\| \leq r\}$, we have $i\left(A, P \cap T_{r}, P\right)=0$. Applying the additivity of the fixed point index, we have

$$
i\left(A,\left(T_{R} \backslash \bar{T}_{r}\right) \cap P, P\right)=i\left(A, T_{R} \cap P, P\right)-i\left(A, \bar{T}_{r} \cap P, P\right)=1 .
$$

Hence, equation (4.1) has at least one positive solution.

\section{The application for the coupled system of elliptic partial} differential equations. We study the following system of elliptic partial differential equations

$$
\left\{\begin{array}{l}
L u=f(x, v) \quad \text { in } \Omega, \\
L v=g(x, u) \quad \text { in } \Omega, \\
B u=0, B v=0 \quad \text { on } \partial \Omega,
\end{array}\right.
$$

where $\Omega$ is a bounded convex open domain in $R^{n}$ and $\partial \Omega \in C^{2+\mu}$ with $0<\mu<1$, and $L$ is a uniformly elliptic operator in $\Omega$ and defined as follows:

$$
L u=-\sum_{i, j=1}^{n} a_{i j}(x) \frac{\partial^{2} u}{\partial x_{i} \partial x_{j}}+\sum_{i=1}^{n} b_{i}(x) \frac{\partial u}{\partial x_{i}}+c(x) u,
$$

where $a_{i j}(x)=a_{j i}(x), a_{i j}(x), b_{i}(x), c(x) \in C^{\mu}(\bar{\Omega})$ and $c(x) \geq 0$. There exists a constant $\lambda>0$ such that

$$
\sum_{i, j=1}^{n} a_{i j}(x) \xi_{i} \xi_{j} \geq \lambda|\xi|^{2}, \quad \text { for all } x \in \bar{\Omega}
$$

where $\xi=\left(\xi_{1}, \xi_{2}, \ldots, \xi_{n}\right) \in R^{n}$. $B$ is the boundary operator and has the following form:

$$
B u=b(x) u+d \frac{\partial u}{\partial \nu},
$$

where $b(x) \in C^{1+\mu}(\partial \Omega)$ and $\nu \in C^{1+\mu}$ is an outward vector of $\partial \Omega$, and suppose that one of the following occurs:

(i) $d=0$ and $b(x) \equiv 1$;

(ii) $d=1$ and $b(x) \equiv 0$; 
(iii) $d=1$ and $b(x)>0$.

Let $f(x, v): \bar{\Omega} \times R^{+} \rightarrow R^{+}$and $g(x, u): \bar{\Omega} \times R^{+} \rightarrow R^{+}$be continuous, $f(x, 0) \equiv 0, g(x, 0) \equiv 0$. We suppose that

$$
\begin{array}{ll}
\left(A_{1}\right) \quad \lim _{v \rightarrow+\infty} \sup _{x \in \Omega} \frac{f(x, v)}{v^{\alpha}}=0, & \lim _{u \rightarrow+\infty} \sup _{x \in \Omega} \frac{g(x, u)}{u^{\beta}}=0, \\
\left(A_{2}\right) \quad \lim _{v \rightarrow 0^{+}} \inf _{x \in \Omega} \frac{f(x, v)}{v^{\alpha_{2}}}=\infty, & \lim _{u \rightarrow 0^{+}} \inf _{x \in \Omega} \frac{g(x, u)}{u^{\beta_{2}}}=\infty,
\end{array}
$$

where $\alpha$ and $\beta$ are defined by $\left(H_{1}\right), \alpha_{2}$ and $\beta_{2}$ are defined by $\left(H_{3}\right)$ in Section 4.

Remark 5.1. In order to study the existence of solutions of (5.1), we consider the boundary value problem of the linear elliptic partial differential equation

$$
\begin{cases}L u(x)=v(x) & x \in \Omega, \\ B u(x)=0 & x \in \partial \Omega .\end{cases}
$$

For every $v \in C^{\mu}(\bar{\Omega})$, we denote by $K v$ the unique solution of the linear boundary value problem (5.2). Let $(K v)(x)=u_{v}(x), x \in \Omega$, where $K: C^{\mu}(\bar{\Omega}) \rightarrow C^{2+\mu}(\bar{\Omega})$ is a linear completely continuous operator, for details see [1]. For the nonlinear elliptic boundary value problem (5.1), we define the Nemytskii operators

$$
\left(F_{1} v\right)(x)=f(x, v(x)), \quad\left(F_{2} u\right)(x)=g(x, u(x)), \quad x \in \bar{\Omega} .
$$

Remark 5.2. In Section 4, let $E=C(\bar{\Omega})$ and $P=\{u \in C(\Omega): u(x) \geq$ 0 , for all $x \in \bar{\Omega}\}$; Theorem 4.1 and Theorem 4.2 still hold.

Theorem 5.3. Suppose that the conditions $\left(A_{1}\right)$ and $\left(A_{2}\right)$ are satisfied. Then (5.1) has at least one positive solution $(u, v) \in C^{2}(\bar{\Omega}) \times C^{2}(\bar{\Omega})$.

Proof. It follows from Remark 5.1 that $(u, v) \in C^{2}(\bar{\Omega}) \times C^{2}(\bar{\Omega})$ is the solution of boundary value problem (5.1) if and only if $(u, v) \in$ $C(\bar{\Omega}) \times C(\bar{\Omega})$ is the solution of the system of integral equations

$$
\left\{\begin{array}{l}
u(x)=\int_{\Omega} k_{1}(x, y) f(y, v(y)) d y \\
v(x)=\int_{\Omega} k_{2}(x, y) g(y, u(y)) d y
\end{array}\right.
$$


where $k_{i}(x, y): \Omega \times \Omega \rightarrow R^{+}, i=1,2$, are Green functions. It is evident that the nonlinear integral equations system (5.3) is equivalent to the following nonlinear double integral equation of Hammerstein type:

$$
u(x)=\int_{\Omega} k_{1}(x, y) f\left(y, \int_{\Omega} k_{2}(y, z) g(z, u(z)) d z\right) d y
$$

Let

$$
A u(x)=\int_{\Omega} k_{1}(x, y) f\left(y, \int_{\Omega} k_{2}(y, z) g(z, u(z)) d z\right) d y
$$

By Remark 5.1, we have $A=K_{1} F_{1} K_{2} F_{2}$, and the maximum principle (e.g., [16]) implies that $K_{1}$ and $K_{2}$ are positive linear operators. It is easily seen that $A: P \rightarrow P$ is completely continuous from (5.5) and Remark 5.1. Next we show that the operator $A$ has at least one fixed point. By condition $\left(A_{1}\right)$, there exist $\alpha, \beta \in(0,1)$ and $a_{1}(x) \geq 0$, $a_{2}(x) \geq 0, b_{1}(x) \geq 0, b_{2}(x) \geq 0$ such that:

$$
\begin{aligned}
& f(x, v) \leq a_{1}(x) v^{\alpha}+b_{1}(x), \quad \text { for all } x \in \Omega, v \geq 0 \\
& g(x, u) \leq a_{2}(x) u^{\beta}+b_{2}(x), \quad \text { for all } x \in \Omega, u \geq 0
\end{aligned}
$$

By Step 1 of the proof of Theorem 4.1 and Remark 5.2, we have that $i\left(A, P \cap T_{R}, P\right)=1$.

It follows from $\left(A_{2}\right)$ that there exist $\alpha_{2} \in(0,+\infty), \alpha_{2} \beta_{2} \in(0,1)$, $m_{1}(x) \geq 0, m_{2}(x) \geq 0$ and sufficiently small $r>0$ and $s \in(0, r)$ such that

$$
\begin{aligned}
& f(x, v) \geq m_{1}(x) v^{\alpha_{2}}, \quad \text { for all } x \in \Omega, 0 \leq v \leq s, \\
& g(x, u) \geq m_{2}(x) u^{\beta_{2}}, \quad \text { for all } x \in \Omega, 0 \leq u \leq r .
\end{aligned}
$$

Let $T_{r}=\{u \in E \mid\|u\| \leq r\}$. Since $g(z, 0) \equiv 0$ and the continuity of $g(z, u)$, we have

$$
\int_{\Omega} k_{2}(y, z) g(z, u(z)) d z \leq s, \quad \text { for all } 0 \leq u \leq r, y \in \Omega
$$


Therefore, we have

$$
\begin{aligned}
A u(x) & =\int_{\Omega} k_{1}(x, y) f\left(y, \int_{\Omega} k_{2}(y, z) g(z, u(z)) d z\right) d y \\
& \geq \int_{\Omega} k_{1}(x, y) m_{1}(y)\left(\int_{\Omega} k_{2}(y, z) g(z, u(z)) d z\right)^{\alpha_{2}} d y \\
& \geq \int_{\Omega} k_{1}(x, y) m_{1}(y)\left(\int_{\Omega} k_{2}(y, z) m_{2}(z) u^{\beta_{2}}(z) d z\right)^{\alpha_{2}} d y \\
& =B_{2} u(x),
\end{aligned}
$$

where

$$
B_{2} u(x)=\int_{\Omega} k_{1}(x, y) m_{1}(y)\left(\int_{\Omega} k_{2}(y, z) m_{2}(z) u^{\beta_{2}}(z) d z\right)^{\alpha_{2}} d y .
$$

It is easily seen that $B_{2}: P \rightarrow P$ is an $\alpha_{2} \beta_{2}$ homogeneous operator. From [1, Lemma 5.3], we have $K_{1}$ and $K_{2}$ are linear completely continuous operators and e-positive. By Definition 2.2, there exist $\alpha>0, e \in P \backslash\{\theta\}$ such that

$$
\begin{aligned}
B_{2} e & =\int_{\Omega} k_{1}(x, y) m_{1}(y)\left(\int_{\Omega} k_{2}(y, z) m_{2}(z) e^{\beta_{2}} d z\right)^{\alpha_{2}} d y \\
& =\int_{\Omega} k_{1}(x, y) v^{*} d y \\
& \geq \alpha e
\end{aligned}
$$

where

$$
v^{*}=m_{1}(y)\left(\int_{\Omega} k_{2}(y, z) m_{2}(z) e^{\beta_{2}}(z) d z\right)^{\alpha_{2}} \not \equiv 0 .
$$

For condition $\left(H_{5}\right)$ of Theorem 4.2, let $\alpha=\delta$. By (5.6), we have $B_{2} e \geq \delta e$. The conditions $\left(H_{1}\right),\left(H_{3}\right)$ and $\left(H_{5}\right)$ are satisfied. We have $i\left(A, P \cap T_{r}, P\right)=0$. Therefore, equation (5.1) has at least one positive solution $(u, v) \in C^{2}(\bar{\Omega}) \times C^{2}(\bar{\Omega})$.

\section{REFERENCES}

1. H. Amann, On the number of solutions of nonlinear equtions in ordered Banach spaces, J. Funct. Anal. 14 (1972), 925-935.

2. Fixed point equations and nonlinear eigenvalue problems in ordered Banach spaces, SIAM Rev. 18 (1976), 620-709. 
3. M. Chhetri and P. Girg, Existence and nonexistence of positive solutions for a class of superlinear semipositone systems, Nonlin. Anal. 71 (2009), 4984-4996.

4. K. Deimling, Nonlinear functional analysis, Springer-Verlag, Berlin, 1985.

5. L.H. Erbe and H.Y. Wang, On the existence of positive solutions of ordinary differential equations, Proc. Amer. Math. Soc. 120 (1994), 743-748.

6. D.J. Guo and V. Lakshmikantham, Nonlinear problems in abstract cones, Academic Press, Boston, 1988.

7. A. Hammerstein, Nichtlineare Integralgleichungen nebst Anwendungen, Acta Math. 54 (1929), 117-176.

8. L. Hu and L.L. Wang, Multiple positive solutions of boundary value problems for systems of nonlinear second-order differential equations, J. Math. Anal. Appl. 335 (2007), 1052-1060.

9. G. Infante and P. Pietramala, Existence and multiplicity of non-negative solutions for systems of perturbed Hammerstein integral equations, Nonlin. Anal. 71 (2009), 1301-1310.

10. K.Q. Lan, Multiple positive solutions of singular Hammerstein integral equations and applications to periodic boundary value problems, Appl. Math. Comput. 154 (2004), 531-542.

11. H.Y. Li and J.X. Sun, Positive solutions of boundary value problem for a system of nonlinear ordinary differential equations, Ann. Diff. Equat. 21 (2005), $153-160$.

12. W.R. Li and B.S. Xu, Convex function-inequality-average, Education Press of Liaoning, Shenyang, 1990 (in Chinese).

13. Z.D. Liang and C.Y. Wang, A theorem on operator equation of positive $\alpha$ homogeneous and its applications, Acta Math. Sin. 39 (1996), 204-208 (in Chinese).

14. R.Y. Ma, Multiple nonnegative solutions of second-order systems of boundary value problems, Nonlin. Anal. 42 (2000), 1003-1010.

15. E. Neumann, Inequalities involving multivariate convex functions III, Rocky Mountain J. Math. 42 (2012), 251-256.

16. M.H. Protter and H.F. Weinberger, Maximum principles in differential equations, Prentice-Hall, Englewood Cliffs, NJ, 1967.

17. J.X. Sun, Nonlinear functional analysis and its application, Science Press, Beijing, 2007 (in chinese).

18. J.X. Sun and X.Y. Liu, Computation for topolpgical degree and its applications, J. Math. Anal. Appl. 202 (1996), 785-796.

19. J.G. Wang, Existence of nontrivial solutions to nonlinear systems of Hammerstein integral equations and applications, Indian J. Pure Appl. Math. 31 (2001), 1303-1311.

20. Z.L. Yang and D.O'Regan, Positive solvability of systems of nonlinear Hammerstein integral equations, J. Math. Anal. Appl. 311 (2005), 600-614. 
School of Science, Xi'an Polytechnic University, Xi'an, Shannxi, 710048, P.R. CHINA

Email address: xinghui1230@126.com

Department of Mathematics, Jiangsu Normal University, Xuzhou, Jiangsu, 221116, P.R. CHINA

Email address: jxsun7083@163.com 